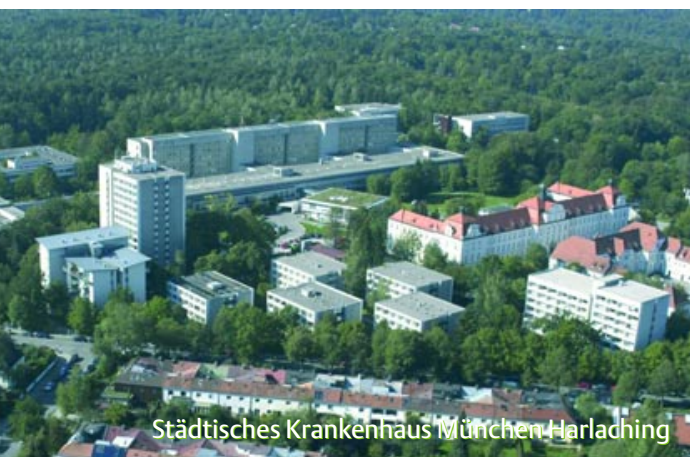

B ayern, mit 12,4 Millionen Einwohnern größtes Bundesland Deutschlands, verfügt derzeit (Stand Oktober 2004) über 17 Palliativstationen an Krankenhäusern mit insgesamt 172 Betten, neun stationäre Hospize mit 78 Betten, etwa 90 Hospizvereine und Hospizinitiativen sowie vier Akademien für Palliativmedizin, Palliativpflege und Hospizarbeit als Institutionen der Aus-, Fort- und Weiterbildung in Palliativmedizin, Palliativpflege und anderen psycho-sozialen Berufsgruppen. Ein Universitätslehrstuhl für Palliativmedizin ist in Planung. Gemessen an den übrigen Bundesländern nimmt Bayern bezüglich der Bettenzahl pro einer Million Einwohner bei den Palliativstationen einen oberen Rang ein. Im Bereich stationärer Hospize liegt es jedoch im bundesweiten Vergleich gemeinsam mit Thüringen und Rheinland-Pfalz auf den letzten drei Plätzen (Abb. 1).

Für ganz Deutschland bezeichnend ist der Mangel an palliativmedizinischen Konsiliardiensten im stationären und ambulanten Bereich, die zahlenmäßig an den Fingern beider Hände abzuzählen sind. Dies ist besonders im Vergleich zum Mutterland des „palliative care“ Großbritannien - zu sehen, das über etwa 350 palliativmedizinische Kon-

\title{
Aktueller Stand und zukünftige Perspektiven Netzwerk Palliativmedizin in Bayern
}

\author{
R.C. Hartenstein \\ IV. Medizinische Abteilung (Onkologie, Hämatologie, Palliativmedizin) \\ Städtisches Krankenhaus München-Harlaching, München
}

klinikarzt 2005; $34(1+2)$ : 33-36

Bayern nimmt bezüglich Bettenzahl pro einer Million Einwohner im Vergleich zu den übrigen Bundesländern bei den Palliativstationen einen oberen Rang ein, bei den stationären Hospizen liegt es jedoch im unteren Bereich der Rangliste. Bislang bestehen nur vereinzelt palliativmedizinische Konsiliardienste im stationären und ambulanten Bereich. Die Problematik bei der Integration und Vernetzung der Palliativmedizin in Bayern liegt jedoch weniger in den Defiziten bei Hospizen und Konsiliardiensten, sondern in der geografischen Aufteilung palliativmedizinischer und hospizlicher Einrichtungen. Für die nicht so großzügig ausgestatteten Landkreise Bayerns wurden und werden daher Aus-, Fort-, und Weiterbildungsangebote erarbeitet, um qualifizierte, personelle Kapazitäten zum Auf- und Ausbau eines Versorgungsnetzwerkes für Schwerstkranke und Sterbende auch in ländlichen Gebieten Bayerns zu schaffen. Grundlagen zur Qualifizierung sind die neue Approbationsordnung 2002 für Studierende, die neue Weiterbildungsordnung 2003 für die Ärzte in Bayern, Qualifizierungsmaßnahmen zur Schaffung des „palliativmedizinisch kompetenten Arztes“ und die Implementierung von Grundkenntnissen in Palliativpflege in die Ausbildung zur ,palliativpflegerisch kompetenten Pflegekraft“. In Gebieten, in denen Einrichtungen für die Betreuung Schwerstkranker und Sterbender sowie qualifiziertes Personal noch nicht ausreichend zur Verfügung stehen, erfolgt die Umsetzung des Aufbaus eines „Netzwerks Palliativmedizin“ in mehreren aufeinander abgestimmten Schritten.

siliarteams verfügt. Palliativmedizinische Konsiliardienste in Krankenhäusern und im ambulanten Bereich werden zunehmend eine wichtige Beratungs- und Vermittlungsfunktion bei der integrierten palliativmedizinischen Versorgung Schwerstkranker und Sterbender übernehmen.

\section{„Entwicklungsbedarf“ in ländlichen Gebieten}

Die Problematik bei der Integration und Vernetzung der Palliativmedizin in Bayern liegt jedoch weniger in den Defiziten bei Hospizen und Konsiliardiensten. Langsam aber stetig entstehen hier neue Einrichtungen - allerdings fast ausschließlich in oder am Rande von Ballungsgebieten. Bayern ist jedoch ein Flächenstaat mit ausgedehnten ländlichen Bereichen. Besonders der nordostbayerische Raum von Freyung bis Hof und der gesamte mittelbayerische Donauverlauf von Neu-Ulm bis Passau sind palliativmedizinisch absolut unterversorgt (Abb. 2). Insbesondere für diese Regionen galt es daher, neue Konzepte zur bedarfsgerechten, qualitativ fundierten Versorgung durch Palliativmedizin und Hospizbetreuung zu erarbeiten. 


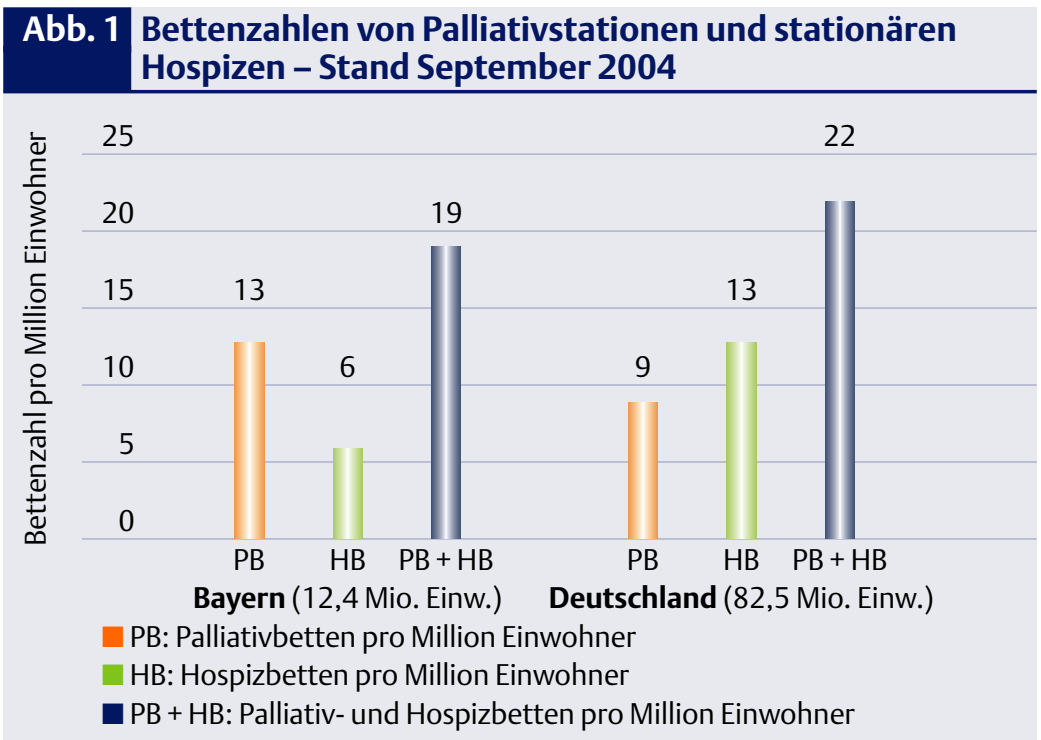

Das Bayerische Staatsministerium für Arbeit, Sozialordnung, Familie und Frauen hat im Dezember 2003 einen „Expertenkreis Palliativmedizin und Hospizarbeit“ gegründet, der derzeit ein umfassendes Konzept der palliativmedizinischen und hospizlichen Versorgung in Bayern erarbeitet. In dem etwa 30-köpfigen Gremium sind alle Organisationen und Initiativen vertreten, die sich mit der Begleitung Schwerstkranker und Sterbender befassen - zum Beispiel die Bayerische Landesärztekammer, die Deutsche Gesellschaft für Palliativmedizin, die Akademien für Palliativmedizin, Palliativpflege und Hospizarbeit, die

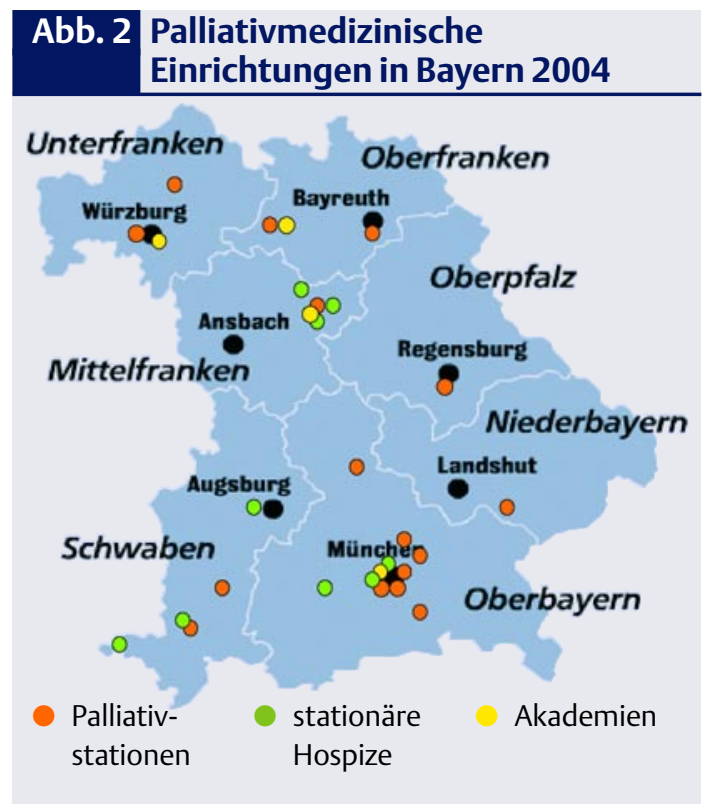

ben. Beispielhaft wird die Vernetzung stationär-ambulanter palliativmedizinischer und hospizlicher Versorgung am Beispiel der Situation im Städtischen Krankenhaus MünchenHarlaching, Akademisches Lehrkrankenhaus der Universität München, dargestellt (Abb. 3).

Wie in vielen Netzwerken ist auch hier der Hausarzt des Patienten in der Funktion als "Lotse“ in die Versorgung eingebunden. Eine Besonderheit in München-Harlaching ist jedoch das so genannte Brückenteam aus einer Palliativpflegekraft und einem Sozialarbeiter. Dieses ist auf der Palliativstation angesiedelt und regelt vorausschauend die Abläufe der Patientenaufnahme auf die Station und der Entlassung in ein betreutes Zuhause oder in ein stationäres Hospiz. Ähnlich ist ein Modellprojekt mit Brückenschwestern an der Palliativstation in Würzburg.

Für die weniger großzügig mit palliativmedizinischen Einrichtungen, Hospizen, ambulanten Diensten und qualifiziertem Personal ausgestatteten Landkreise Bayerns müssen alle Anstrengungen zur Qualifikationen durch Aus-, Fort- und Weiterbildung unternommen werden. Zudem müssen die personellen Kapazitäten geschaffen werden, um den Auf- und Ausbau eines Versorgungsnetzwerks für Schwerstkranke und Sterbende auch in Nicht-Ballungsräumen zu ermöglichen. und -pflegerischer Grundkompetenz aller ambulant und stationär tätigen Ärzte und Pflegekräfte und zur qualifizierten, spezialisierten Beratung und Mitbetreuung durch Palliativmediziner, Palliativpflegekräfte und psychosoziale Fachkräfte erarbeitet.

\section{Ballungsräume sind besser versorgt}

In den städtischen Ballungsräumen München, Nürnberg und Würzburg sind die Voraussetzungen für eine angemessene Versorgungsqualität und Vernetzung dagegen durch die Präsenz von Palliativstationen, palliativmedizinischen Konsiliardiensten, stationären Hospizen, ambulanten Palliativ- sowie Hospizdiensten und deren Ausstattung mit qualifiziertem, multiprofessionellen Personal bereits weit gehend gege-

\section{In Aus- und Weiterbildung integriert}

Die Aus- und Weiterbildungsgänge zur Qualifizierung von Medizinern sind in der neuen Approbationsordnung (AO 2002) und der neuen Weiterbildungsordnung (WBO 2003) festgelegt. Die Approbationsordnung fordert vom Medizinstudenten Kenntnisse im Umgang mit ethischen Fragestellungen sowie Fähigkeiten zur Hilfe und Betreuung bei Schwerstkranken und Sterbenden. Die Prüfungsaufgaben sollen Schmerzbehandlung und Palliativmedizin berücksichtigen. Als erste Universität in Deutschland hat die Universität München die Palliativmedizin als Pflichtlehr- und Prüfungsfach für Medizinstudenten eingeführt. 
Die Weiterbildungsordnung 2003 erkennt die Palliativmedizin als eigenständige Zusatzweiterbildung nach der Facharztanerkennung in Gebieten der unmittelbaren Patientenversorgung an. Hierzu sind eine praktische Weiterbildung von zwölf Monaten bei einem Weiterbildungsbefugten - die anteilig durch 120 Stunden Fallseminare einschließlich Supervision ersetzt werden kann - sowie 40 Stunden Kursweiterbildung in Palliativmedizin erforderlich. Außerdem sind in der Basisweiterbildung aller an der unmittelbaren Patientenversorgung beteiligten Fachgebiete Kenntnisse, Erfahrungen und Fertigkeiten in der Betreuung palliativmedizinisch $\mathrm{zu}$ versorgender Patienten gefordert.

Seit dem 1. August 2004 ist die Weiterbildungsordnung für die Ärzte Bayerns in Kraft. In dieser sind noch Übergangsbestimmungen für den Erwerb der Zusatzbezeichnung für Ärzte integriert, die bei Inkrafttreten der Weiterbildungsordnung berechtigt sind, eine Facharztbezeichnung in einem Gebiet der unmittelbaren Patientenversorgung zu führen oder die 24 Monate Weiterbildung in einem derartigen Gebiet nachweisen. Diese Ärzte werden auf Antrag zur Prüfung zugelassen, wenn sie innerhalb der letzten acht Jahre mindestens zwölf Monate an einer Weiterbildungsstätte oder einer vergleichbaren Einrichtung regelmäßig in der Palliativmedizin tätig waren und dokumentieren können, dass sie die nach der Weiterbildungsordnung für die Zusatzbezeichnung geforderten Kenntnisse, Erfahrungen und Fertigkeiten erworben haben.

Die bayerischen Akademien für Palliativmedizin, Palliativpflege und Hospizarbeit bieten zusätzlich nach den Empfehlungen der Deutschen Gesellschaft für Palliativmedizin Qualifizierungskurse für den Erwerb der Zusatzbezeichnung an. Zu den Qualifizierungsmaßnahmen zur Schaffung des „palliativmedizinisch kompetenten Arztes“ erarbeitet die Bayerische Landesärztekammer derzeit zusammen mit der Münchener Akademie und dem Hausärzteverband eine strukturierte Fortbildung von Hausärzten in Palliativmedizin im Rahmen eines fünfjährigen Curriculums. Interessierte Ärzte können sich in einem 40-stündigen Basiskurs zur Palliativmedizin im Rahmen des Angebots der bayerischen Akademien für Palliativmedizin, Palliativpflege und Hospizarbeit fortbilden. Die Implementierung von Grundkenntnissen in Palliativpflege in die Ausbildung zur ,palliativpflegerisch kompetenten Pflegekraft" soll auf der Grundlage des „Curriculums Palliativpflege 2004“ der Deutschen Gesellschaft für Palliativmedizin erfolgen.

\section{Was ist noch zu tun?}

Bedingt durch die derzeit noch nicht ausreichende Verfügbarkeit von qualifiziertem Fachpersonal vor allem in unterversorgten, zumeist ländlichen Gebieten Bayerns soll der Auf- und Ausbau eines „Netzwerks Palliativmedizin“ in mehreren aufeinander abgestimmten Schritten erfolgen ( Abb. 4):

- Ermittlung und Bündelung der Ressourcen vor Ort ohne Anspruch auf palliativmedizinische oder -pflegerische Qualifikation unter Einschluss von Hausarzt, Fachärzten, Krankenhäusern, ambulanten Pflegediensten, Sozialdiensten, Apotheken, Sanitätshäusern, kirchlichen Gemeinden, kirchlichem Be- suchsdienst und nicht zuletzt von Angehörigen, Freunden und Nachbarschaftshilfe

- gemeinsame Fortbildung („voneinander lernen“) und Aufbau von Beratungsteams aus in der Betreuung von Schwerstkranken und Sterbenden Erfahrenen

- dem Hausarzt kommt eine zentrale Rolle im Netzwerk als Behandelnder, Betreuer, Berater, Vermittler und Wegweiser zu

- Entwicklung von vernetzten Kommunikationsstrukturen und Informationswegen

- palliativmedizinische und -pflegerische Grundkompetenz aller in der Region tätigen Ärzte und Pflegekräfte durch zwischenzeitliche Aus- und Fortbildung

- flächendeckendes, bedarfsgerechtes Angebot an Palliativmedizinern und Palliativpflegekräften, die beratend oder auch mitbehandelnd tätig werden. Schaffung eines Palliativmedizinischen Konsiliardienstes, bestehend aus Palliativmediziner, Palliativpflegekraft und Sozialarbeiter im Netz mit gemeinsamer ambulanter oder stationärer Basis.

Trotz alles Engagements und aller Bemühungen von Medizin und Pflege, beteiligten Verbänden und

\section{Abb. 3 Ambulant-stationäres Netzwerk Palliativmedizin}

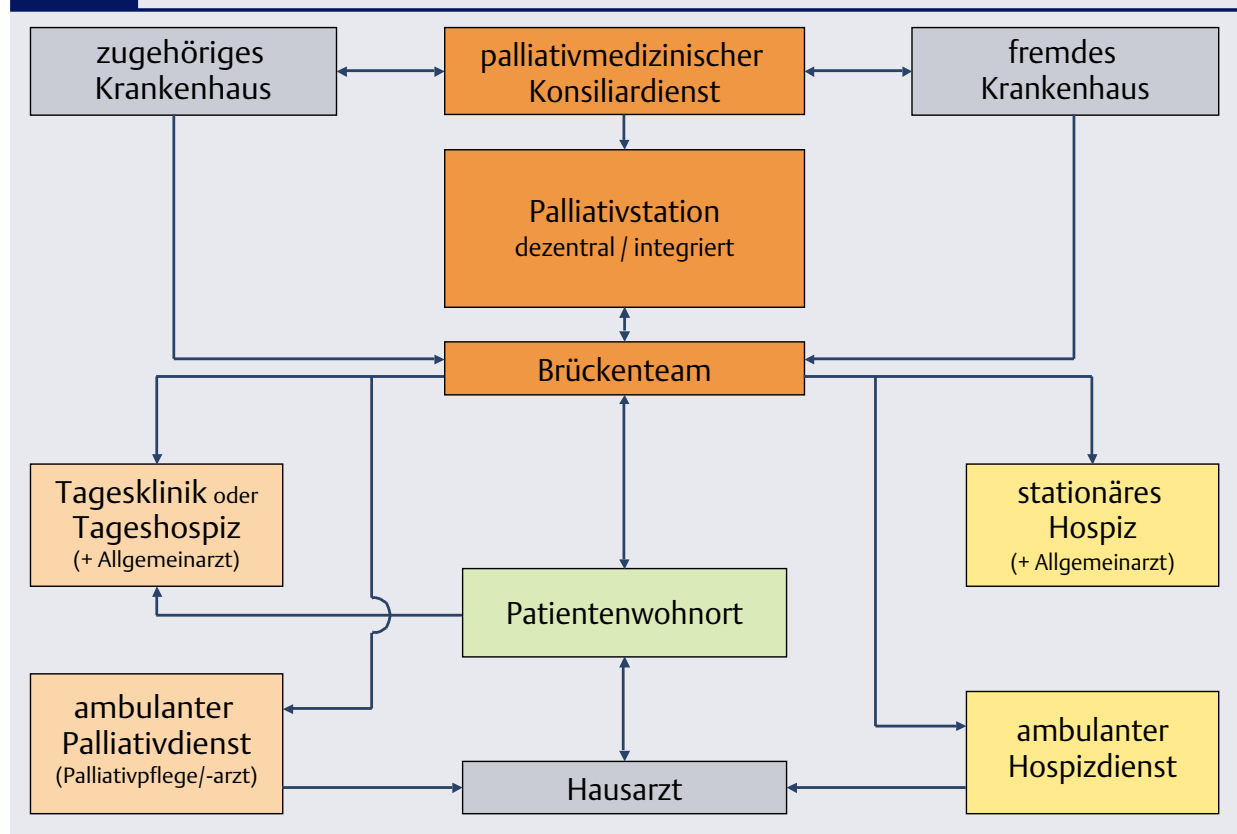




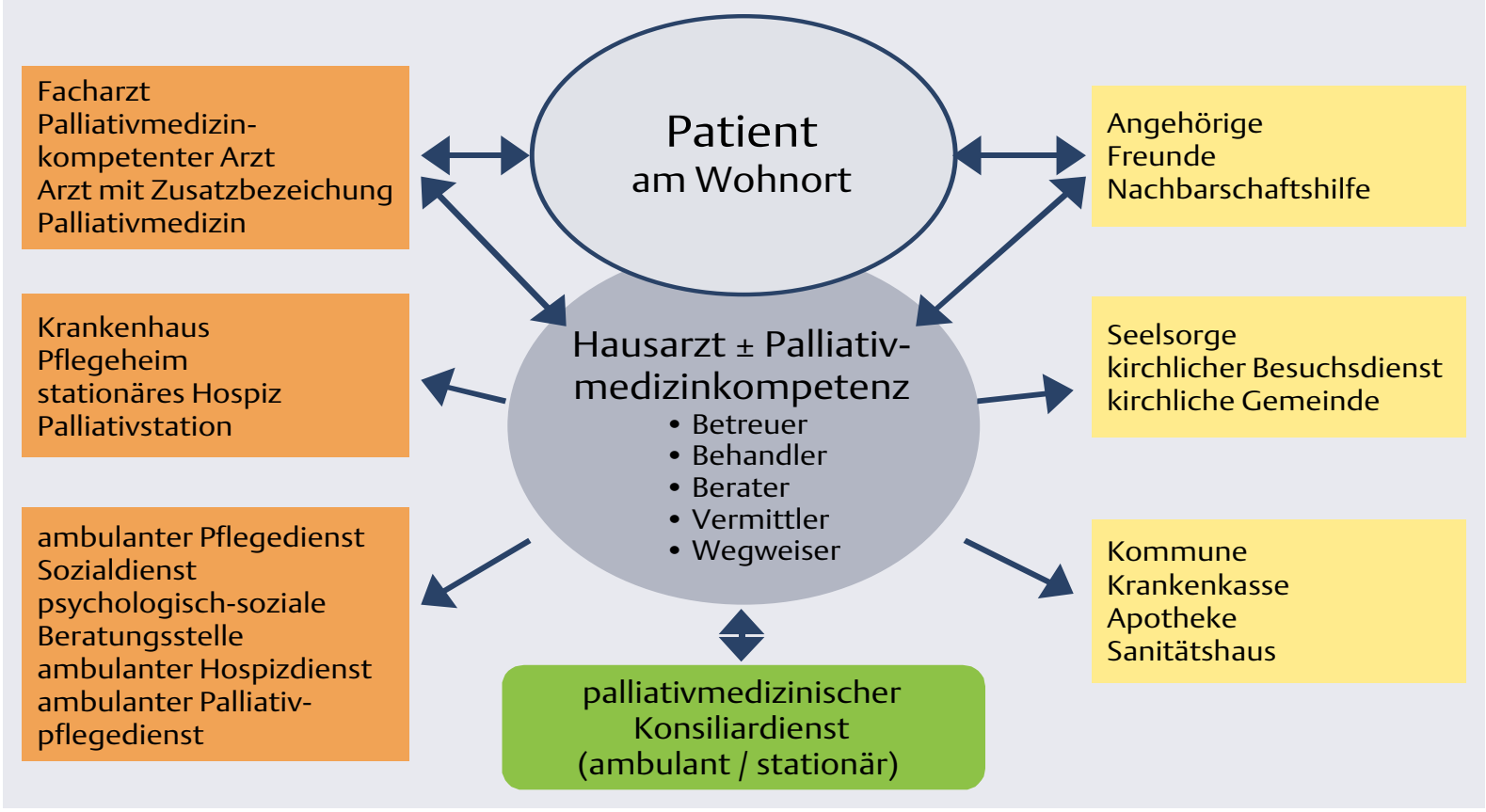

Gesellschaften gemeinsam mit dem Freistaat Bayern eine kompetente, bedürfnisgerechte Betreuung von Schwerstkranken und Sterbenden in allen Landkreisen Bayerns sicherzustellen, verbleiben noch eine Reihe von Fragen und Problemen, deren Beantwortung und Lösung für die Etablierung eines Netzwerks palliativmedizinischer und hospizlicher Versorgung essenziell sind. Dazu zählen

- die zeitnahe Qualifikation von Behandelnden und Betreuern (Aus-, Fort- und Weiterbildung)

- die Schaffung von Ausbildungskapazität (Akademien, palliativmedizinische Einrichtungen)

- die Entwicklung von vernetzten Kommunikationsstrukturen und Informationswegen

- die Finanzierung palliativmedizinischer und palliativpflegerischer Leistungen besonders im ambulanten, aber auch im stationären Bereich

- die sozialrechtliche Absicherung pflegender Angehöriger.

Die sozialrechtliche Absicherung pflegender Angehöriger ist inzwischen sogar per Gesetzestext dokumentiert: „Das Recht des Patienten auf palliativmedizinische Betreuung ist seit dem 09. Juni 1999 gesetzlich verankert. Besondere finanzielle Dispositionen für die Hauspflege werden angekündigt. Jeder Arbeitnehmer hat seit dem 09. Juni 1999 Anrecht auf einen dreimonatigen unbezahlten Urlaub zur Sterbebegleitung von Verwandten ersten Grades und jeder anderen in seinem Haushalt lebenden Person." - Nur leider wurde dieses Gesetz in unserem Nachbarland Frankreich erlassen!

\section{Current Status and Future Perspec- tives - Palliative Medicine Network in Bavaria}

In terms of the number of palliative beds per million of the population, Bavaria ranks high in comparison with other German parts. In the case of hospices, however, it has a low position on the ranking list. To date, only scattered in-hospital and ambulatory palliative medical consulting services are available. The problem with the integration and networking of palliative medicine in Bavaria is, however, not so much deficits with regard to hospices and consulting services, but in the geographical distribution of the palliative-medical facilities and hospices. For the less-well endowed regions in Bavaria, training and further education opportunities have been and will be created and put in place, with the aim of establishing qualified personnel for the establishment and expansion of a management network for the seriously ill and dying patients in the rural areas of Bavaria. The basis for such qualifications are the new (2002) regulations for medical students, the new further education ordinance (2003) for Bavarian physicians, qualification measures aimed at producing physicians with palliative-medical skills, and the implementation of basic knowledge in palliative care in the training of nursing personnel with palliative-medical competence. In areas still lacking sufficient facilities for the care of the very ill and the dying as well as qualified personnel, the setting up of a palliative-medical network will be realised in a number of consecutive coordinated steps.

\section{Key Words}

palliative care - network qualification - Bavaria

\section{Anschrift des Verfassers}

Prof. Dr. Reiner C. Hartenstein Präsident der Bayerischen Krebsgesellschaft Nymphenburger Str. 21A 80335 München 